\title{
Blood Oxygen Level-Dependent Imaging and Intravoxel Incoherent Motion MRI of Early Contrast-Induced Acute Kidney Injury in a Rabbit Model
}

\author{
Yongfang Wang $^{a} \quad$ Xin Zhang ${ }^{a} \quad Z^{2}$ hengxu Hua ${ }^{a}$ Lizhi Xie $^{b} \quad$ Xuan Jiang ${ }^{c}$ \\ Rongjia Wang ${ }^{\text {a }}$ Peirong Gao ${ }^{d}$ Ke Ren $^{\mathrm{e}}$ \\ a Department of Radiology, First Hospital of China Medical University, Shenyang, China; \\ ${ }^{b}$ GE Healthcare, MR Research China, Beijing, China; ${ }^{c}$ Cardiac Surgery, First Hospital of \\ China Medical University, Shenyang, China; ${ }^{d}$ Department of Ultrasound, First Hospital of

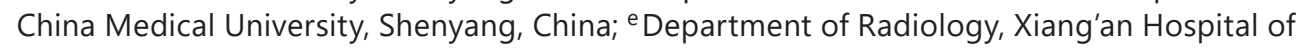 \\ Xiamen University, Xiamen, China
}

\section{Keywords}

Contrast-induced acute kidney injury · Intravoxel incoherent motion · Blood oxygenation level-dependent imaging · Hypoxia-inducible factor-1 $\alpha \cdot$ Vascular endothelial growth factor

\begin{abstract}
Background: To evaluate the application of blood oxygenation level-dependent (BOLD) imaging and intravoxel incoherent motion (IVIM) magnetic resonance imaging (MRI) on assessing early contrast-induced acute kidney injury (CIAKI). Materials: Sixty rabbits were randomly chosen to undergo iohexol (1.0, 2.5, and $5.0[\mathrm{gl} / \mathrm{kg}]$, respectively; $n=15$ for each group) or saline injection ( $n=15)$. In each group, 6 rabbits underwent MRI at $24 \mathrm{~h}$ before injection and after injection of iohexol or saline ( $1 \mathrm{~h}$ and 1,2,3, and 4 days); meanwhile, out of the remaining 9 rabbits, 3 were chosen for MRI acquisition, and then they were killed at specific time points ( $1 \mathrm{~h}, 1$ day, and 3 days, respectively). Results: The strong attenuation of pure molecular diffusion (D), apparent diffusion coefficient (ADC), and perfusion fraction $(f)$ was observed at 1 day, while pseudodiffusion coefficient $\left(D^{*}\right)$ showed a significant decrease at $1 \mathrm{~h}$ after iohexol injection. A distinct elevation of apparent transverse relaxation rate (R2*) reached the maximum levels on day 1 , which was consistent with the expression of hypoxia-inducible factor $-1 \alpha$ and vascular endothelial growth factor. ADC, D, and R2* correlated well with histopathological parameters and biochemical parameters. Conclusion: BOLD combined with IVIM is effective to monitor renal pathophysiology associated with CIAKI.
\end{abstract}




\section{Kidney \\ Blood Pressure \\ Research}

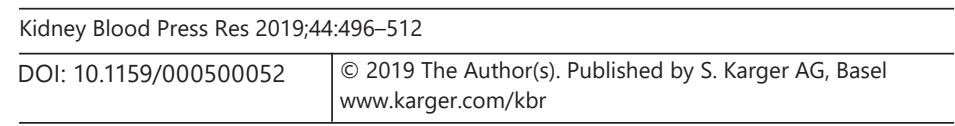

Wang et al.: fMRI and Contrast-Induced Acute Kidney Injury

\section{Introduction}

Contrast media (CM) has been increasingly applied in clinical practice to assist in the acquisition of various radiographic imaging modes and complex interventional procedures $[1,2]$. Nevertheless, the invasiveness of CM may induce contrast-induced acute kidney injury (CIAKI), which is the most prevalent cause of acute kidney injury [3]. The underlying mechanisms leading to CIAKI have not been fully revealed, despite the extensive studies on clinical disease assessment and therapy response [4].

Multiple noninvasive imaging techniques open a new era for the estimation of renal microcirculation and pathophysiology in vivo, such as blood oxygenation level-dependent (BOLD) imaging and intravoxel incoherent motion (IVIM) magnetic resonance imaging (MRI) [5-7]. BOLD is able to reflect the oxygenation status in tissues via the variation of the magnetic signal with respect to changes in blood deoxygenated $\mathrm{Hb}$ concentration $[8,9]$. IVIM-MRI evaluates the microscopic motion as two distinct diffusion constants: the perfusion-related parameters (pseudodiffusion constant $\mathrm{D}^{*}$, perfusion fraction $\mathrm{f}$ ) and the molecular diffusionrelated parameters (apparent diffusion coefficient [ADC], the true random water diffusion $\mathrm{D}$ ) [10]. To be more specific, decreased renal perfusion and renal medullary hypoxia are recognized as factors predisposing to the onset of CIAKI [11]. It may be desirable to combine BOLD with IVIM in terms of detecting the renal damage provoked by CM. As reported, the rabbit model is a suitable substitute for studying CIAKI with regard to renal changes, because they can be rendered in healthy rabbits with a single injection of CM without additional renal insults $[12,13]$. On the other hand, larger kidneys could avoid limitations on spatial resolution and signal-to-noise ratio with 3T MRI.

The current study aimed to examine the application of BOLD and IVIM in quantitative analysis of renal functional impairment in CIAKI. The CM toxicity was also investigated by injecting different doses and verifying the dose response with iohexol. Furthermore, the occurrence of CIAKI would be determined by serum creatinine (Scr), while the renal tubular injury would be confirmed using urinary neutrophil gelatinase-associated lipocalin (UNGAL). Cellular hypoxia would be evidently measured by examining the distribution of hypoxiainducible factor- $1 \alpha$ (HIF-1 $\alpha$ ) and vascular endothelial growth factor (VEGF).

\section{Materials and Methods}

\section{Subject Selection}

Sixty sexually mature male New Zealand white rabbits aged 9-10 weeks and weighing 2.5-3.0 kg were included. The rabbits had free access to standard food and tap water until $8 \mathrm{~h}$ prior to MRI examination. They were anesthetized via a venous cannula (24 gauge) inserted in the marginal ear vein with $3 \%$ pentobarbital sodium at a dose of $0.5 \mathrm{~mL} / \mathrm{kg}$. After baseline functional MRI (fMRI) scan, prewarmed $\left(37^{\circ} \mathrm{C}\right.$ ) iohexol (GE Healthcare) or saline was injected into the marginal ear vein.

\section{fMRI Protocol}

After 30 min of anesthesia, all the rabbits underwent MR scan on a 3.0 T Twin Speed whole-body MR scanner (General Electric Medical Systems, Milwaukee, WI, USA) using a cardiac matrix coil with 8 independent receiver elements. In order to minimize the motion artifact of kidney, the rabbits were placed in the supine position with a belly band firmly bound on the abdomen. BOLD and IVIM images were independently acquired by 2 professional radiologists in a double-blind manner. fMRI parameters are shown in Table 1. 
Kidney

Blood Pressure

Research
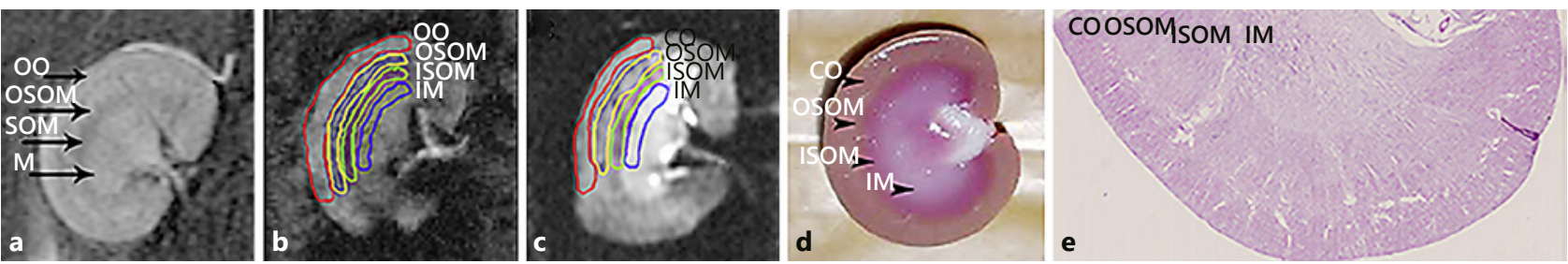

Fig. 1. Different regions in the rabbit kidney and regions of interest (ROIs). a T2 image. b R2* image and ROIs. c ADC image and ROIs $\left(b=0 \mathrm{~s} / \mathrm{mm}^{2}\right)$. d Resected specimen. e A histological slice. The ROIs were drawn in the cortex (CO), outer stripe of outer medulla (OSOM), inner stripe of outer medulla (ISOM), and inner medulla (IM).

Table 1. BOLD and IVIM technical parameters

\begin{tabular}{lll}
\hline & BOLD & IVIM \\
\hline Sequence type & 2 D-GRE & DW-EPI \\
Slices, $n$ & 5 & 5 \\
Section thickness, mm & 3.0 & 3.0 \\
Repetition time, ms & 96.2 & 4,050 \\
Echo time, ms & $4.4-48.7$ & 95.5 \\
Orientation & Coronal & Coronal \\
Bandwidth, Hz/pixel & 41.67 & - \\
\hline Field of view, cm ${ }^{2}$ & $16.0 \times 16.0$ & $16.0 \times 16.0$ \\
Matrix & $256 \times 256$ & $160 \times 160$ \\
Excitations, $n$ & 2.0 & 2.0 \\
Acquisition time & 4 min $10 \mathrm{~s}$ & 6 min $41 \mathrm{~s}$ \\
b-values, s/mm ${ }^{2}$ & - & $0,10,20,50,100,200,400,600,800,1,200$ \\
Flip angle & $30^{\circ}$ & - \\
Breathing protocol & Free breathing & Free breathing \\
\hline
\end{tabular}

\section{fMRI Data Analysis}

IVIM-MRI data were calculated as described by Le Bihan et al. [14]; the relationship between signal intensities and $b$ values is as follows:

$$
S_{b} / S_{0}=(1-f) \times \exp (-b \times D)+f \times \exp \left(-b \times D^{*}\right)
$$

where $S_{b}$ is the signal intensity at a specific $b$ value, and $S_{0}$ is the signal intensity at a b value of 0 , $D$ represents the true diffusion, $\mathrm{D}^{*}$ the pseudodiffusion coefficient, $\mathrm{f}$ the perfusion fraction, and $b$ the diffusion gradient. Since $D^{*}$ is greater than $D$, the influence of $D^{*}$ can be neglected $\left(>200 \mathrm{~s} / \mathrm{mm}^{2}\right)$. Ultimately, the previous equation can be simplified as follows:

$$
S_{b} / S_{0}=(1-f) \times \exp (-b \times D)
$$

Thus, D was calculated according to Eq. (2); subsequently, fand D* were calculated based on Eq. (1).

Parametric images of fMRI were analyzed with the ADVANCE Workstation software (General Electric Medical Systems). According to the study of Niles et al. [15], fMRI images were segmented into four regions corresponding to the anatomic compartments of renal tissues, including cortex (CO), outer stripe of the outer medulla (OSOM), inner stripe of the 


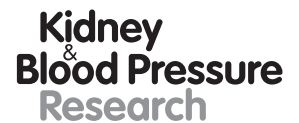

Kidney
Blood Pressure
Research

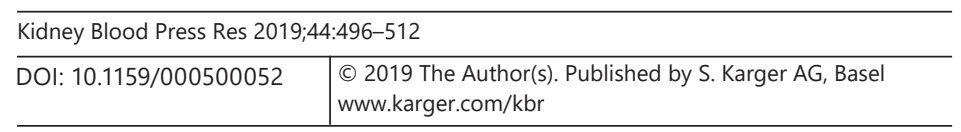

Wang et al.: fMRI and Contrast-Induced Acute Kidney Injury

outer medulla (ISOM), and inner medulla (IM) (Fig. 1). In each kidney, regions of interest were manually placed by a segmentation of the coronal BOLD and IVIM images with the same shape and size (approx. 35-45 $\mathrm{mm}^{2}$ ) (Fig. 1). Additionally, the quantitative regional R2*, ADC/D, and $D^{*} / f$ measurements were performed three times by the same radiologists, independently.

\section{Role of the Administered CM in the Kidney}

Sixty rabbits were administered intravenous CM at dosages of 1.0 (group 1), 2.5 (group 2 ), or 5.0 g (group 3) iodine/ $\mathrm{kg}$ body weight, respectively, and group 4 was injected with saline ( $n=15$ for each group) $[12,16,17]$. In each group, 6 rabbits underwent fMRI scan at baseline (before injection), 1 h, 1 day, 2 days, 3 days, and 4 days after injection; 9 rabbits were randomly selected for histopathological examination after fMRI at each respective time point ( 1 h, 1 day, and 3 days; $n=3$ for each time point).

\section{Histological Analysis}

The right kidneys were immediately placed and fixed in 4\% paraformaldehyde for $72 \mathrm{~h}$, subsequently dehydrated and embedded in paraffin, and eventually sections were cut at $5 \mu \mathrm{m}$ for hematoxylin-eosin staining. Two pathologists with more than 5 years of experience independently analyzed the sections. Histopathological changes were assessed for tubular desquamation, necrosis, tubular collapse, cytoplasmic vacuoles, and glomerular atrophy. Severity of tissue damage was semiquantitatively analyzed by assigning a score of $0-4$ to each histopathological change: 0 , no damage; 1 , minimal injury (less than 25\%); 2 , moderate injury (between 25 and 50\%); 3, intermediate injury (between 50 and 75\%); and 4, severe injury (more than $75 \%)$ [18].

\section{Evaluation of Potential Kidney Injury}

Briefly, after deparaffinization and antigen retrieval, the slides were blocked with $3 \%$ hydrogen peroxide for $20 \mathrm{~min}$, and then protein blocking solution was used to inhibit nonspecific bindings for $30 \mathrm{~min}$. Sequentially, the specimens were incubated overnight at $4{ }^{\circ} \mathrm{C}$ with the primary antibody HIF-1 $\alpha$ (dilution 1:100) (Novus, NB100-105, USA) and polyclonal mouse anti-rabbit VEGF antibody (dilution 1:500) (Novus, NBP2-45235, USA). Thereafter, the tissue sections were incubated for $30 \mathrm{~min}$ at room temperature with biotin-labeled goat anti-mouse IgG secondary antibody (dilution 1:200) (Beyotime, A0216, Shanghai, China). The HIF- $1 \alpha$ and VEGF expression were obtained using the ImageJ software.

\section{Evaluation of Renal Function}

Blood (1.5-2.0 mL) was collected via ear vein prior to MRI examination and $1 \mathrm{~h}, 1$ day, and 3 days after the injection of iohexol/saline. The samples were centrifuged at 3,200 rpm $\left(4{ }^{\circ} \mathrm{C}\right)$ for $20 \mathrm{~min}$ to obtain serum. The concentration of the Scr was analyzed in our local clinical laboratory.

Urine samples (1.0-1.5 mL) were collected at baseline and $1 \mathrm{~h}, 1$ day, and 3 days after $\mathrm{CM}$ administration in each group. At the time of assessment, urinary biomarker was examined using rabbit NGAL ELISA kits (fk-bf2464; Yuanmu Biological Technology Co., Ltd.) according to the manufacturer's instructions. In order to minimize the confounding effects of the urine flow rate, urine creatinine concentrations were analyzed by the local clinical laboratory [19]. The concentrations of urine NGAL were tested at various time points, with urine creatinine examined at same time, correspondingly.

\section{Statistical Analysis}

The results are presented as mean \pm SD. Statistical analyses were performed using SPSS version 22.0. One-way analysis of variance (ANOVA) with the LSD test was used for inter- 


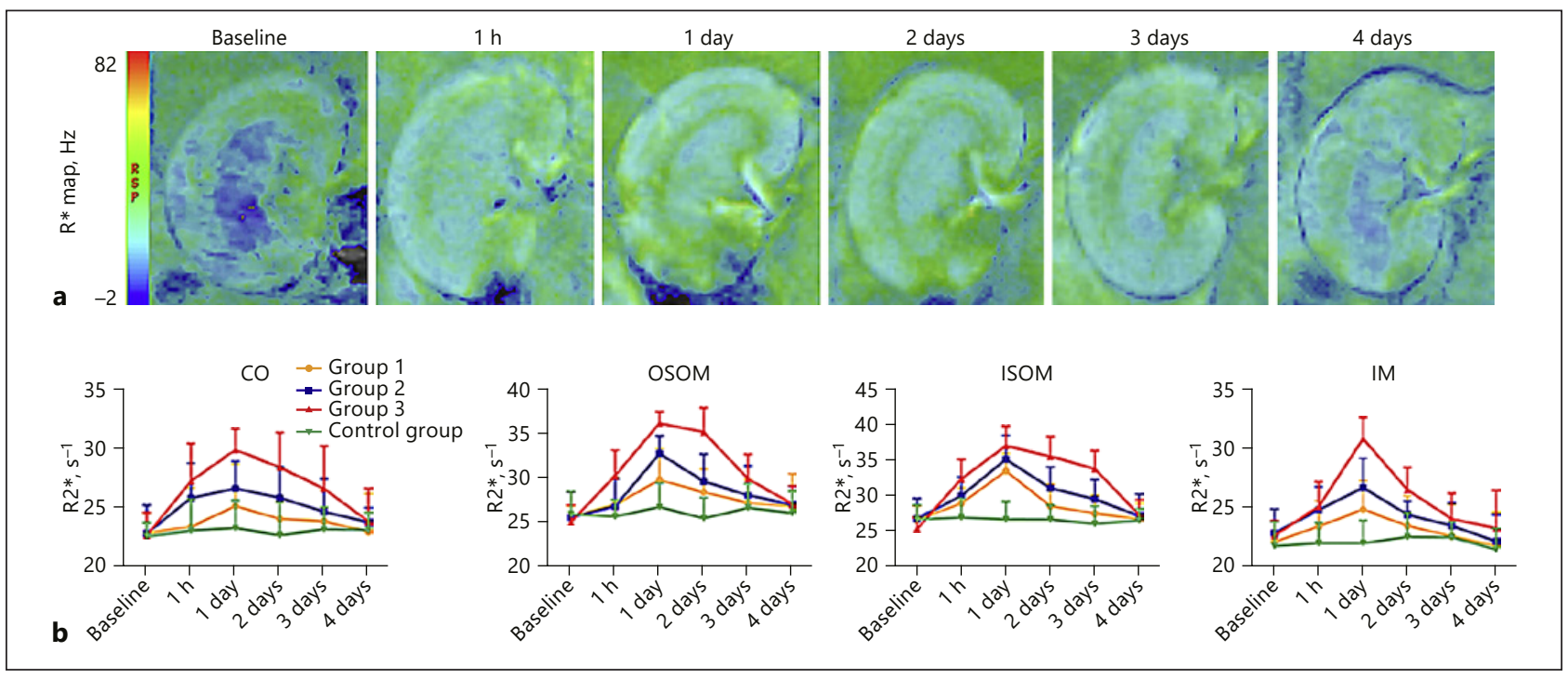

Fig. 2. Representative $\mathrm{R} 2 *$ of a rabbit kidney from group 3 and the time course of $\mathrm{R} 2 *$ changes in the four groups. a Representative R2* images in group 3. All maps are demonstrated on the same window and level settings. The intensity of ISOM was greater than the remaining regions, which implied lower level of oxygenation. $\mathbf{b}$ The time course of R2* changes in the four groups. Maximum R2* signal changes appeared on day 1, and then gradually recovered towards the baseline.

Table 2. Summary of mean values of R2* $\left(\mathrm{s}^{-1}\right)$, ADC $\left(\times 10^{-4} \mathrm{~mm}^{2} / \mathrm{s}\right) / \mathrm{D}\left(\times 10^{-4} \mathrm{~mm}^{2} / \mathrm{s}\right)$, and $\mathrm{D}^{*}\left(\times 10^{-3} \mathrm{~mm}^{2} / \mathrm{s}\right) / \mathrm{f}(\%)$ in group 3 (rabbits subjected to CIAKI) at different time points

\begin{tabular}{|c|c|c|c|c|c|c|c|}
\hline $\begin{array}{l}\text { Kidney } \\
\text { tissue }\end{array}$ & Treatment & Baseline & $1 \mathrm{~h}$ & 1 day & 2 days & 3 days & 4 days \\
\hline $\mathrm{CO}$ & $\begin{array}{l}\mathrm{R}^{*} \\
\mathrm{ADC} \\
\mathrm{D} \\
\mathrm{D}^{*} \\
\mathrm{f}\end{array}$ & $\begin{array}{r}22.74 \pm 1.95 \\
10.03 \pm 0.45 \\
4.12 \pm 0.22 \\
10.57 \pm 0.52 \\
43.23 \pm 1.90\end{array}$ & $\begin{array}{c}27.32 \pm 3.15 \\
9.03 \pm 0.25 \\
3.64 \pm 0.20 \\
8.55 \pm 0.25^{*} \\
38.13 \pm 1.34^{*}\end{array}$ & $\begin{array}{r}29.95 \pm 1.75^{*} \\
8.51 \pm 0.42^{*} \\
2.99 \pm 0.24^{*} \\
9.10 \pm 0.74^{*} \\
36.05 \pm 1.36^{*}\end{array}$ & $\begin{array}{c}28.48 \pm 2.90 \\
8.89 \pm 0.16 \\
3.43 \pm 0.26^{*} \\
9.25 \pm 0.66 \\
40.55 \pm 2.12\end{array}$ & $\begin{array}{r}26.68 \pm 3.58 \\
9.67 \pm 0.23 \\
3.71 \pm 0.24 \\
9.56 \pm 0.39 \\
42.33 \pm 1.89\end{array}$ & $\begin{array}{r}23.92 \pm 2.78 \\
10.13 \pm 0.29 \\
3.91 \pm 0.18 \\
9.89 \pm 0.21 \\
43.08 \pm 2.29\end{array}$ \\
\hline OSOM & $\begin{array}{l}\mathrm{R}^{*} \\
\mathrm{ADC} \\
\mathrm{D} \\
\mathrm{D}^{*} \\
\mathrm{f}\end{array}$ & $\begin{array}{r}25.22 \pm 1.92 \\
9.71 \pm 0.23 \\
4.12 \pm 0.26 \\
9.19 \pm 0.24 \\
41.57 \pm 1.19 \\
\end{array}$ & $\begin{array}{r}30.41 \pm 2.85 \\
9.14 \pm 0.39 \\
3.55 \pm 0.33 \\
7.96 \pm 0.18^{*} \\
36.53 \pm 1.85^{*}\end{array}$ & $\begin{array}{r}36.29 \pm 1.29^{*} \\
8.70 \pm 0.24^{*} \\
3.17 \pm 0.16^{*} \\
8.32 \pm 0.18^{*} \\
34.58 \pm 1.82^{*}\end{array}$ & $\begin{array}{c}35.30 \pm 2.70^{*} \\
9.06 \pm 0.16^{*} \\
3.48 \pm 0.21^{*} \\
8.49 \pm 0.32 \\
36.85 \pm 2.43\end{array}$ & $\begin{array}{r}30.06 \pm 2.75 \\
9.61 \pm 0.38 \\
3.65 \pm 0.21 \\
8.64 \pm 0.20 \\
39.62 \pm 2.03 \\
\end{array}$ & $\begin{array}{r}27.23 \pm 2.02 \\
9.89 \pm 0.25 \\
3.91 \pm 0.37 \\
8.96 \pm 0.15 \\
40.70 \pm 1.62 \\
\end{array}$ \\
\hline ISOM & $\begin{array}{l}\mathrm{R} 2 * \\
\mathrm{ADC} \\
\mathrm{D} \\
\mathrm{D}^{*} \\
\mathrm{f}\end{array}$ & $\begin{array}{r}25.49 \pm 1.62 \\
9.44 \pm 0.24 \\
3.96 \pm 0.21 \\
9.20 \pm 0.25 \\
40.50 \pm 1.56\end{array}$ & $\begin{array}{c}32.51 \pm 2.71 \\
8.54 \pm 0.38^{*} \\
3.40 \pm 0.21 \\
7.90 \pm 0.18^{*} \\
34.83 \pm 1.33^{*}\end{array}$ & $\begin{array}{r}37.16 \pm 2.71^{*} \\
8.00 \pm 0.50^{*} \\
2.91 \pm 0.34^{*} \\
8.33 \pm 0.29^{*} \\
33.47 \pm 1.97^{*}\end{array}$ & $\begin{array}{c}35.67 \pm 2.74^{*} \\
8.21 \pm 0.34^{*} \\
3.21 \pm 0.22^{*} \\
8.44 \pm 0.25 \\
37.82 \pm 1.37\end{array}$ & $\begin{array}{c}33.93 \pm 2.57^{*} \\
8.68 \pm 0.38^{*} \\
3.38 \pm 0.28 \\
8.63 \pm 0.13 \\
39.08 \pm 1.57\end{array}$ & $\begin{array}{r}27.45 \pm 2.11 \\
9.16 \pm 0.38 \\
3.83 \pm 0.12 \\
8.91 \pm 0.22 \\
39.82 \pm 1.26\end{array}$ \\
\hline IM & $\begin{array}{l}\mathrm{R} 2^{*} \\
\mathrm{ADC} \\
\mathrm{D} \\
\mathrm{D}^{*} \\
\mathrm{f}\end{array}$ & $\begin{array}{r}22.75 \pm 1.25 \\
11.87 \pm 0.58 \\
5.06 \pm 0.18 \\
8.84 \pm 0.16 \\
40.93 \pm 2.14\end{array}$ & $\begin{array}{c}25.17 \pm 2.12 \\
9.97 \pm 0.50 \\
4.67 \pm 0.30 \\
8.19 \pm 0.14^{*} \\
37.63 \pm 1.22\end{array}$ & $\begin{array}{r}30.92 \pm 1.76^{*} \\
9.61 \pm 0.58^{*} \\
4.21 \pm 0.31^{*} \\
8.28 \pm 0.11^{*} \\
34.98 \pm 1.52^{*}\end{array}$ & $\begin{array}{r}26.61 \pm 1.89 \\
9.84 \pm 0.58 \\
4.57 \pm 0.28 \\
8.33 \pm 0.34 \\
38.48 \pm 2.03\end{array}$ & $\begin{array}{r}24.15 \pm 2.16 \\
10.81 \pm 0.52 \\
4.79 \pm 0.24 \\
8.44 \pm 0.21 \\
40.42 \pm 1.08\end{array}$ & $\begin{array}{r}23.35 \pm 3.22 \\
11.01 \pm 0.66 \\
4.90 \pm 0.25 \\
8.60 \pm 0.27 \\
40.82 \pm 0.99\end{array}$ \\
\hline
\end{tabular}

\footnotetext{
$* p<0.05$ vs. baseline.
} 
Kidney

Blood Pressure

Research
Kidney Blood Press Res 2019;44:496-512

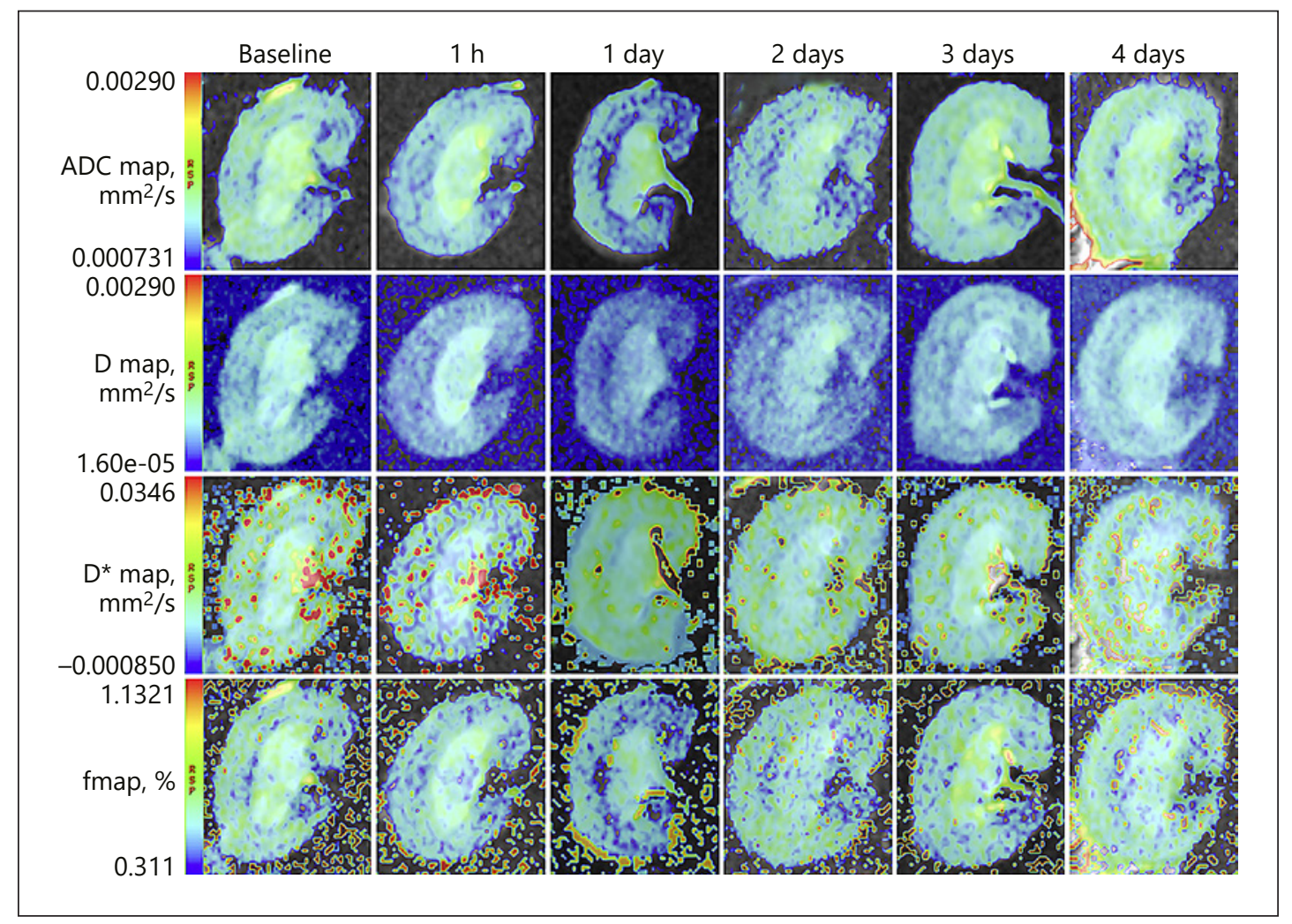

Fig. 3. Representative $A D C / D$, and $D^{*} / f$ maps of a rabbit kidney from group 3 (baseline, 1 h, 1 day, 2 days, 3 days, and 4 days). The cortical and medullary ADC, $\mathrm{D}$, and $\mathrm{f}$ in the kidney decreased significantly on day 1 ; maximum $\mathrm{D}^{*}$ signal changes appeared at $1 \mathrm{~h}$.

group comparisons of fMRI parameters. The repeated ANOVA test was used to compare the baseline values with those after contrast medium injection within a group. Spearman's correlation analysis was employed to assess the relationship between fMRI variables and histological changes or urinary NGAL (uNGAL). $p<0.05$ was considered statistically significant.

\section{Results}

\section{fMRI Measurements in Rabbit Kidneys after CM}

As demonstrated in Figure 2a, iohexol induced a significant increase in R2* in group 3 (rabbits subjected to CIAKI). In groups $1-3$, compared with baseline values, R2* values dramatically increased at $1 \mathrm{~h}$ and up to the maximum levels at 1 day in $\mathrm{CO}(p>0.05, p>0.05$, and $p=0.040$, respectively), OSOM ( $p>0.05, p>0.05$, and $p=0.003$, respectively), ISOM ( $p=$ $0.043, p=0.008$, and $p=0.008$, respectively), and IM ( $p>0.05, p>0.05$, and $p=0.001$, respectively), and subsequently R2* returned to the baseline level within 2,2 , and 4 days, respectively (Fig. 2b).

As demonstrated in Table 2, iohexol induced a marked and significant reduction in ADC/D and $\mathrm{D}^{*} / \mathrm{f}$ in all of the anatomical compartments in group 3 (rabbits subjected to CIAKI), and the IVIM images obtained at each time point are shown in Figure 3.

For renal diffusivity, compared with baseline values, ADC dramatically decreased at $1 \mathrm{~h}$, reaching the bottom on day 1 in $\mathrm{CO}(p>0.05, p=0.003$, and $p=0.002$, respectively), OSOM 


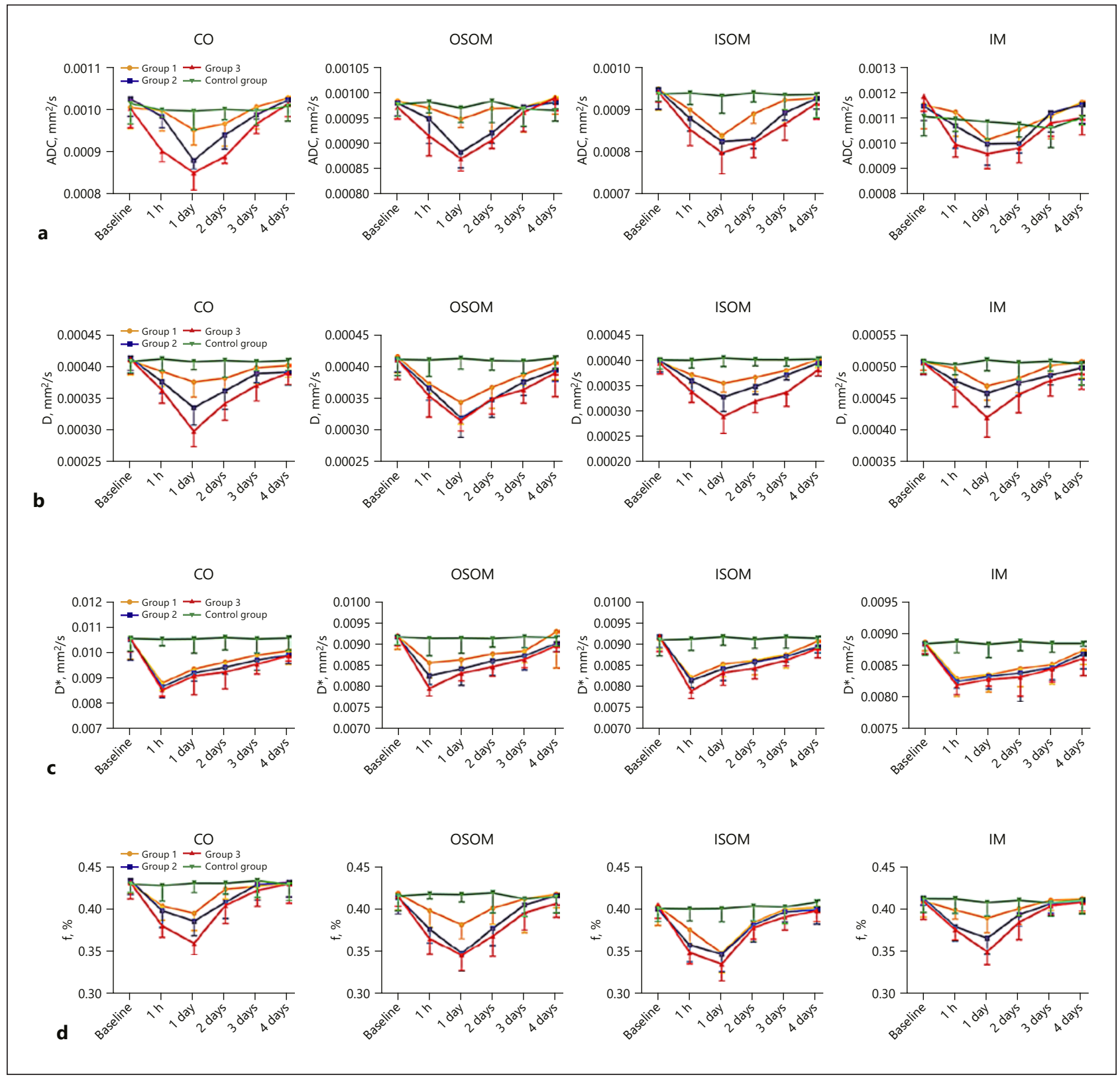

Fig. 4. Summary of temporal changes in $A D C / D$, and $D^{*} / f$ measurements over time in the four groups. a ADC time curves. b $\mathrm{D}$ time curves. $\mathbf{c} \mathrm{D}^{*}$ time curves. $\mathbf{d} \mathrm{f}$ time curves.

( $p>0.05, p=0.048$, and $p=0.015$, respectively), ISOM ( $p=0.046, p=0.027$, and $p=0.014$, respectively), and IM ( $p>0.05, p>0.05, p=0.024$, respectively) in groups $1-3$, and subsequently ADC returned to the baseline value on days 2,3 , and 4, respectively (Fig. 4a).

A similar rapid initial decrease was observed for $\mathrm{D}$, reaching the bottom on day 1 after iohexol administration in groups $1-3$ in $\mathrm{CO}(p>0.05, p=0.037$, and $p=0.015$, respectively), OSOM ( $p>0.05, p=0.031$, and $p=0.012$, respectively), and IM ( $p>0.05, p>0.05$, and $p=$ 0.007 , respectively); in addition, a significant reduction of D in ISOM was observed on day 1 ( $p>0.05, p=0.020, p=0.016$, respectively), followed by a rebound towards the baseline within 0,3 , and 3 days, respectively (Fig. 4 b). 
Kidney
Blood Pressure

Research
Kidney Blood Press Res 2019;44:496-512

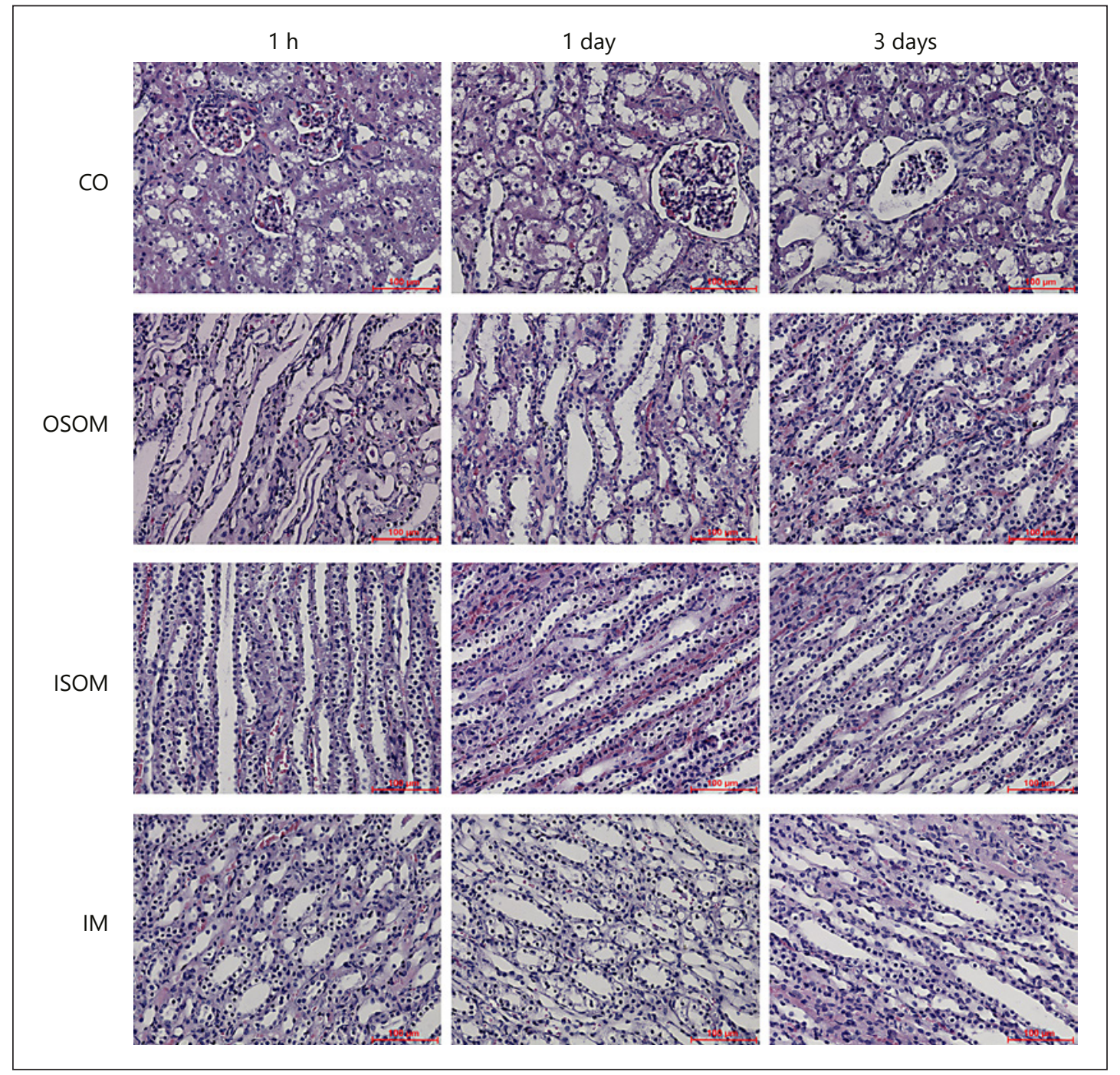

Fig. 5. Hematoxylin-eosin-stained histological changes of the kidneys in group 3 . At $1 \mathrm{~h}$, the proximal tubular epithelial cells became swollen, with pronounced tubular dilatation and intraluminal desquamation; on day 1 , obvious tubular cell vacuolization-necrosis in the proximal tubules was detected; on day 3 , interstitial vasodilation started to become visible, and atrophy in the glomerulus and tubular vacuolization were observed. Scale bar, $100 \mu \mathrm{m}$.

A significant reduction was observed for the perfusion parameter $\mathrm{D}^{*}$ in all of the anatomic compartments in an early stage of contrast-induced renal damage. For groups 1-3, while compared with the baseline values, iohexol decreased $D^{*}$ values to minimum levels at $1 \mathrm{~h}$ in CO ( $p>0.05, p>0.05, p=0.006$, respectively), OSOM ( $p=0.006, p=0.010, p=0.009$, respectively), ISOM ( $p=0.047, p=0.004, p=0.004$, respectively), and IM ( $p=0.041, p=0.007$, and $p=0.029$, respectively), and returned towards the baseline level during a follow-up of 2 days, 2 days, and 2 days, respectively (Fig. 4c).

A similar but slighter change in $\mathrm{f}$ values was detected, where the mean $\mathrm{f}$ value decreased at $1 \mathrm{~h}$, reached its minimum on day 1 in $\mathrm{CO}(p>0.05, p=0.010$, and $p=0.016$, respectively), OSOM ( $p>0.05, p<0.001$, and $p=0.019$, respectively), ISOM ( $p=0.028, p=0.025$, and $p=$ 0.001 , respectively), and IM ( $p>0.05, p=0.014$, and $p=0.034$, respectively), and gradually returned to baseline within 2 days in groups 1-3 (Fig. 4d). 


\section{Kidney \\ Blood Pressure Research}

\begin{tabular}{l}
\hline Kidney Blood Press Res 2019;44:496-512 \\
\hline DOI: 10.1159/000500052
\end{tabular} \begin{tabular}{l}
$\begin{array}{l}\text { ○ 2019 The Author(s). Published by S. Karger AG, Basel } \\
\text { www.karger.com/kbr }\end{array}$ \\
\hline
\end{tabular}

Wang et al.: fMRI and Contrast-Induced Acute Kidney Injury

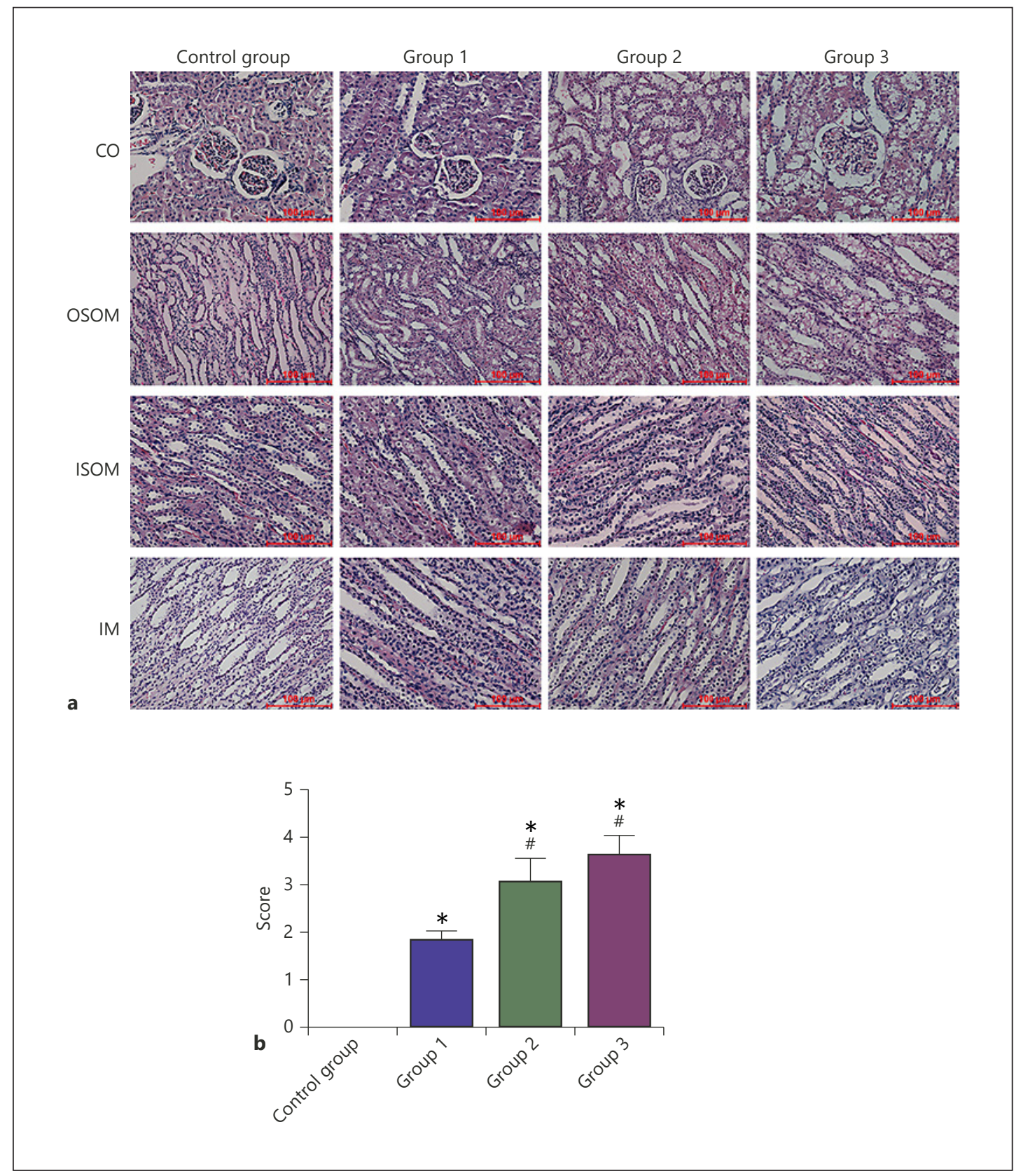

Fig. 6. Hematoxylin-eosin-stains showing histological changes of the kidneys in the four groups on day 1. a Renal histopathological changes. A marked severe injury was observed in group 3. Control group had no renal injury. Scale bar, $100 \mu \mathrm{m}$. b Histological injury scores. ${ }^{*} p<0.05$ vs. control group; ${ }^{\#} p<0.05$ vs. group 1 .

\section{Histological Analysis}

Renal injury occurred at different levels of severity, depending on the injection dosage. Vacuole formation is recognized as the earliest sign of CIAKI, as a result of pinocytosis and lysosomal fusion. Widespread tubular karyopyknosis or necrosis was the predominant change in CIAKI. Pronounced tubular dilatation, atrophy, and fibrosis in the glomerulus, and tubular desquamation could also be observed (Fig. 5). The severity score varied significantly in groups 1-3 (Fig. 6). 
Kidney
Blood Pressure

Research
Kidney Blood Press Res 2019;44:496-512

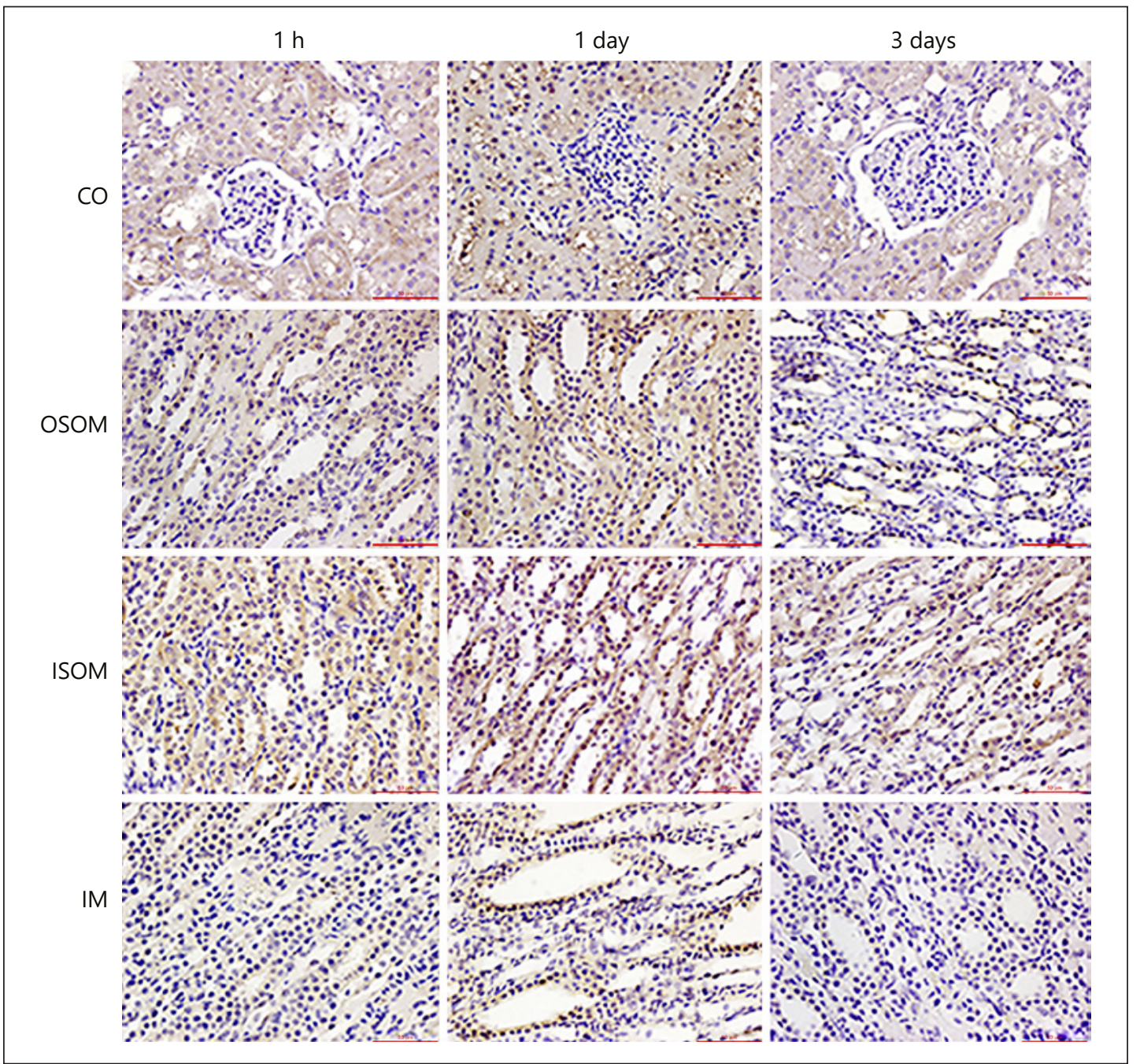

Fig. 7. HIF- $1 \alpha$ signals changes in four renal zones during a long time period in group 3. A marked nuclear accumulation of HIF-1 $\alpha$ occurred largely at day 1 . HIF-1 $\alpha$-positive cells were likely to be observed in the OSOM and ISOM, and low levels of HIF-1 $\alpha$ expression was observed in the IM. Scale bar, $50 \mu \mathrm{m}$.

\section{HIF-1 $\alpha$ and VEGF Immunohistochemistry Results}

HIF-1 $\alpha$ was mainly expressed in the nucleus of tubular cells. After iohexol injection, HIF- $1 \alpha$ was transiently upregulated at $1 \mathrm{~h}$ and confined to a period of time, within 3 days after the induction of the hypoxic insult (Fig. 7). Group 1 and group 2 showed less expression of HIF-1 $\alpha$ than group 3, and slight signal changes were detected in the control group (Fig. 8).

The location of VEGF expression partially corresponded to areas of HIF-1 $\alpha$ induction. Mean VEGF scores were significantly higher in the iohexol-treated groups than in the control group. After injection of iohexol, renal VEGF immunostaining was aggravated along with the increase in iohexol dosages (Fig. 9).

\section{Blood and Urinary Biomarkers}

A dose-dependent rise in Scr was observed in rabbits, when it significantly changed on day 3, whereas no statistically significant change in Scr was observed after iohexol injection at other time points (Fig. 10a). 


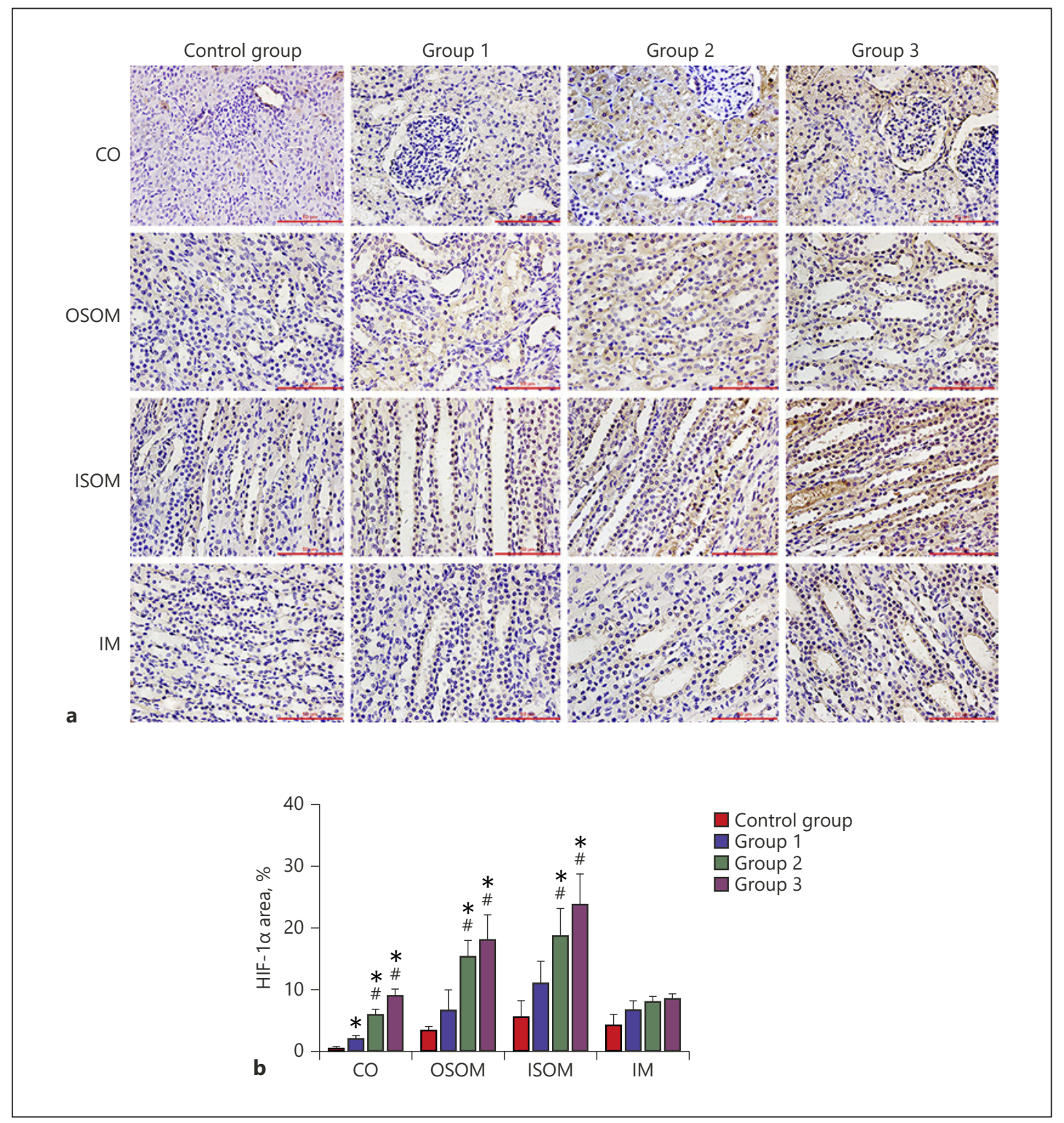

Fig. 8. HIF-1 $\alpha$ intensity changes in the four groups on day 1. a HIF-1 $\alpha$ expression changes in the four groups. A marked nuclear accumulation of HIF-1 $\alpha$ occurred largely in group 3. Scale bar, $50 \mu \mathrm{m}$. b The HIF-1 $\alpha$ score. ${ }^{*} p<0.05$ vs. control group; ${ }^{\#} p<0.05$ vs. group 1 .

Although the concentrations of urine normalized NGAL of groups 1 and 2 increased slightly after contrast, they were not significantly different from the baseline level. For group 3 , the concentration of urine NGAL remained at a comparatively low level at $1 \mathrm{~h}$ and reached the peak at 1 day after the application of contrast medium ( $p=0.032$ vs. baseline) (Fig. 10b).

\section{Correlation between fMRI Parameters and Histological Scores/HIF-1 $\alpha$ Expression/VEGF}

Expression/NGAL

Among all the cases, there was good correlation between histological scores and ADC, D, or $\mathrm{f}$ (Fig. 11a, b, d), while there was fair negative correlation between histological scores and $D^{*}$ values for the medullary kidney (Fig. 11c). 
Kidney
Blood Pressure

Research

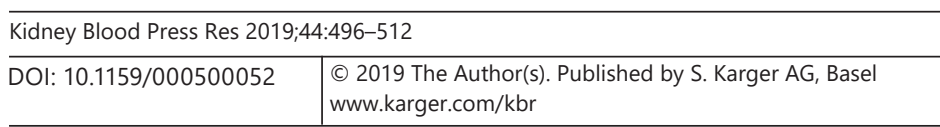

Wang et al.: fMRI and Contrast-Induced Acute Kidney Injury

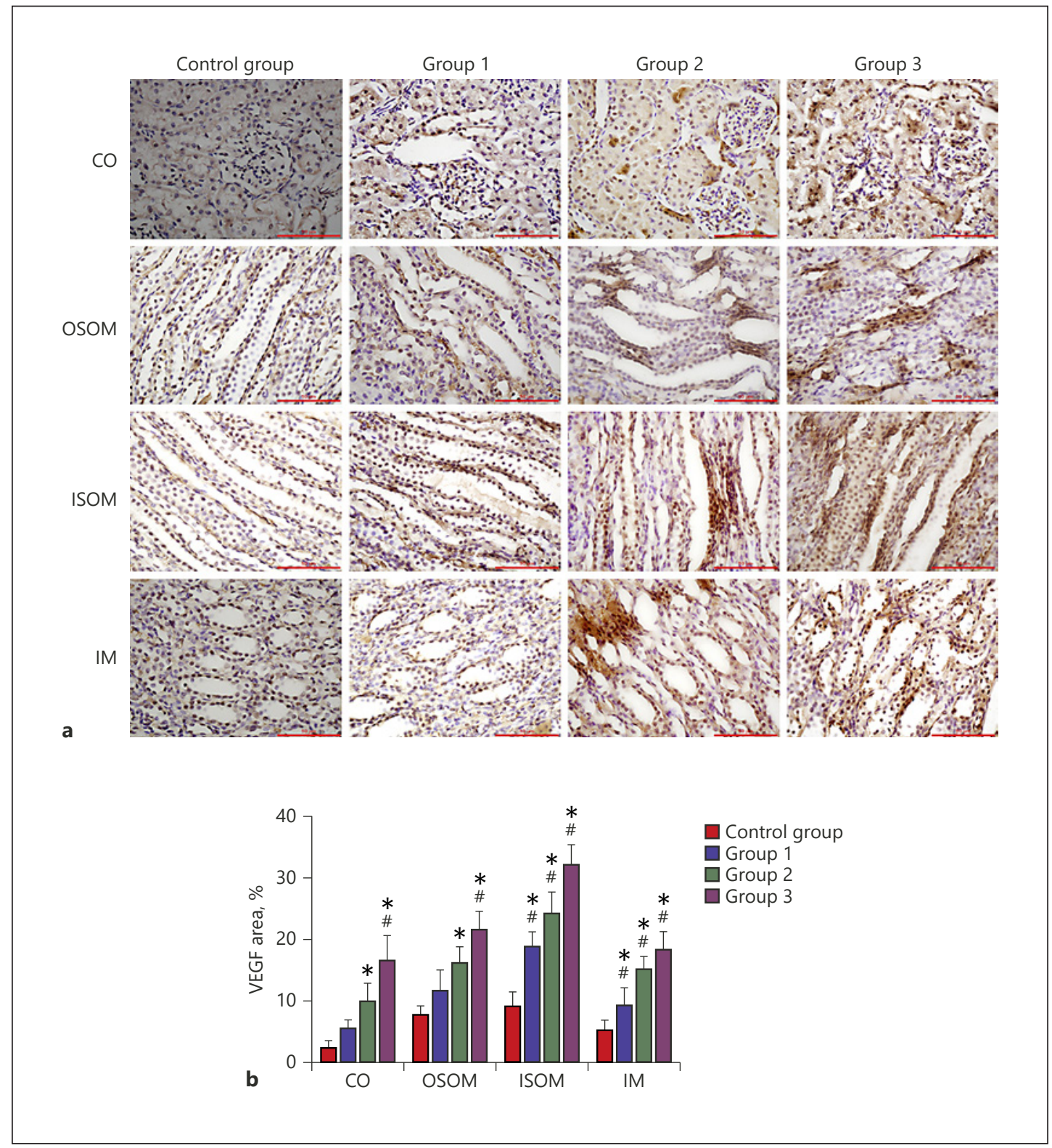

Fig. 9. VEGF intensity changes in the four groups on day 1. a VEGF expression changes in the four groups. A marked VEGF expression was observed in group 3. Scale bar, $50 \mu \mathrm{m}$. b The VEGF score. ${ }^{*} p<0.05$ vs. control group; ${ }^{\#} p<0.05$ vs. group 1.

There was good negative correlation between HIF-1 $\alpha$ expression and ADC, $\mathrm{D}$, or $\mathrm{f}$ values (Fig. 11e, f, h), but poor correlation between HIF-1 $\alpha$ expression and $\mathrm{D}^{*}$ values (Fig. 11g). There was good correlation between uNGAL and ADC or D (Fig. 11i, j) and fair correlation between uNGAL and f (Fig. 11l), while there was no correlation between uNGAL and D* (Fig. 11k).

There was a good correlation between histological scores and R2* values (Fig. 12a). For the medullary kidney, the calculated R2* were significantly positively correlated with HIF-1 $\alpha$ expression, VEGF expression, or NGAL (Fig. 12b-d). 


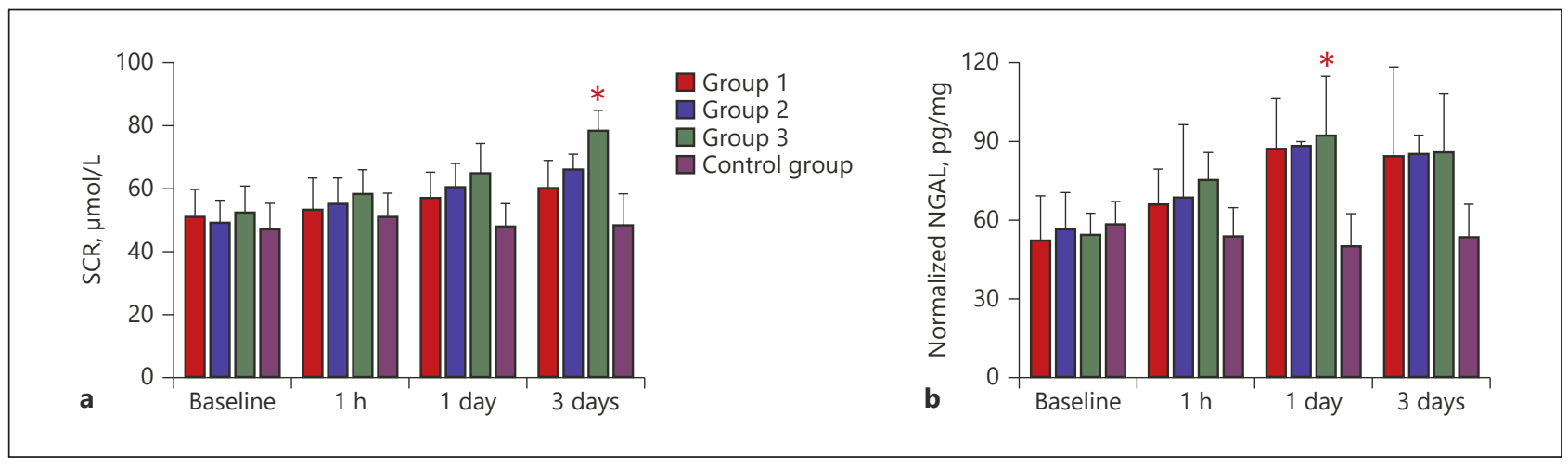

Fig. 10. Serial changes of Scr (a) and NGAL (b) during the acquisition period ( $n=3)$. The histogram demonstrated Scr and NGAL values before and after contrast medium administration. The Scr and NGAL windows of opportunity to predict contrast-induced acute kidney injury were 1 day and 3 days, respectively. A significant difference $(* p<0.05)$ was observed vs. baseline.

\section{Discussion}

In the present study, the value of multiparametric fMRI technologies, i.e. BOLD combined with IVIM, was demonstrated on estimating rabbit renal insufficiency caused by contrast medium in vivo. In addition, New Zealand white rabbits served as good models for understanding the causes leading to CIAKI [20]. The impact of contrast medium dosages on modulating renal vascular and tubular function was achieved by using fMRI, renal histology, HIF-1 $\alpha$, and VEGF as indicators of renal damage.

In this study, we observed that the D value showed a correlation with HIF-1 $\alpha$ in the kidney, where hypoxia was partially attributed to induced tubular injury, in agreement with the theoretical relation described by Rosenberger [21]. Furthermore, tubular injury was correlated with intrarenal diffusion as well [22], which can be confirmed by the good correlation between D and NGAL. NGAL, as a viable tubular damage marker, is rapidly and massively generated in tubule cells of the kidney after renal ischemia reperfusion, released into the urine within hours [23]. An additional explanation for tubular injury may be related to direct cytotoxicity effects or increased level of apoptosis in tubule cells and the successive aggravation of diffusion deficiency [23]. On the other hand, it is known that the intracellular versus extracellular fluid fraction affects renal diffusion [24]. Iohexol displays hyperosmolality and accumulates in the tubular system with a much larger concentration, which will induce prolonged cellular dehydration, eventually leading to a relative reduction in the intrarenal water content.

Our results revealed that the reduction of renal perfusion occurred earlier and was more severely affected than tissue diffusivity with respect to renal dysfunction, which is consistent with Liang et al. [25]. Noticeably, the results also revealed that $\mathrm{f}$ required a time window (i.e., 1-2 days) to recover to the baseline level in all of the four renal regions. Hence, those effects far outweigh the presence of hyperfiltration compensatory mechanism, where arterial pressure and tubular flow were increased [26]. It was hypothesized that an increase in the D* value on day 1 might be associated with vasodilation, while the f value on day 2 might indicate an elevated vascular fluid volume.

In this rabbit model, renal oxygenation was reduced after iohexol injection, as demonstrated using BOLD, where R2* derived from BOLD were strongly correlated with HIF-1 $\alpha$ expression or VEGF expression. It has been proposed that medullary hypoxia, in particular, is a hallmark of CIAKI [27]. One possible explanation is that renal blood flow elevates rapidly, followed by a more prolonged decrease, eventually leading to intrarenal hypoxia after the administration of CM [28]. 


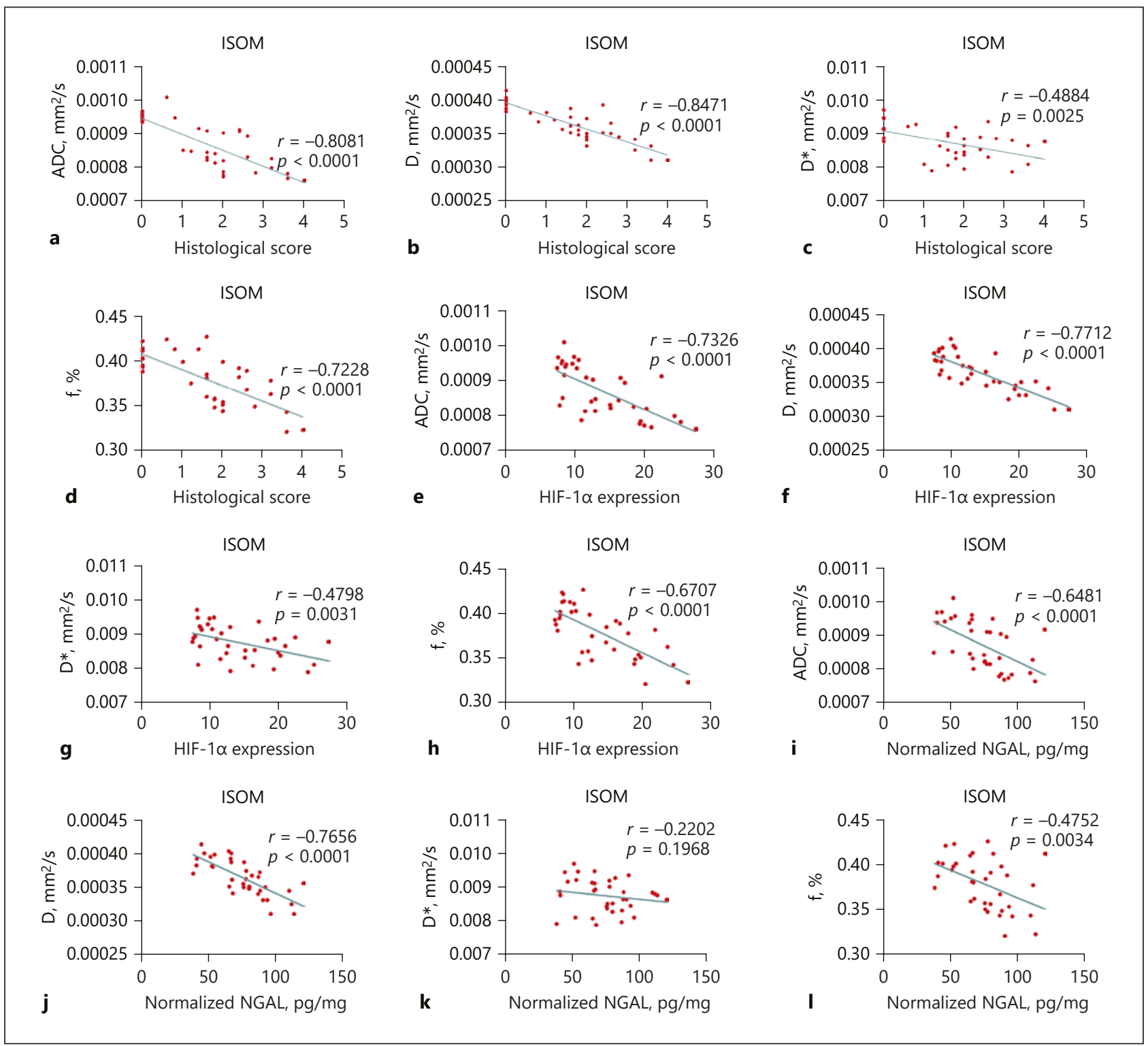

Fig. 11. Correlation analysis for all the measured IVIM parameters with pathology scores. a-d Correlation between histological scores and ADC, D, D*, or f. e-h Correlation between HIF-1 $\alpha$ and ADC, D, D*, or f. i-I Correlation between NGAL and ADC, D, D*, or f.

This hypothesis was supported by our results with the observed reduction in $\mathrm{D}^{*}$ associated with net decreased blood flow. Also, CM applied to the lumen led to constriction and enhanced vasa recta responses to angiotensin II in a series of in vitro studies from rats and humans [29]. In addition, the kidney may increase tubular transport to maintain fluid homeostasis, which results in raised oxygen consumption and a subsequent increase in R2* [30].

The results of our study suggest that iohexol caused a dose response in the whole renal hypoxia and a remarkable reduction in molecular diffusivity and microcapillary perfusion. The previous finding of $\mathrm{Li}$ et al. [31] also showed that renal medullary hypoxia was dose dependent after the application of iodixanol. CM acts in a time- and concentration-dependent manner; an increased dosage may increase CM's cytotoxicity and prolong the time renal 


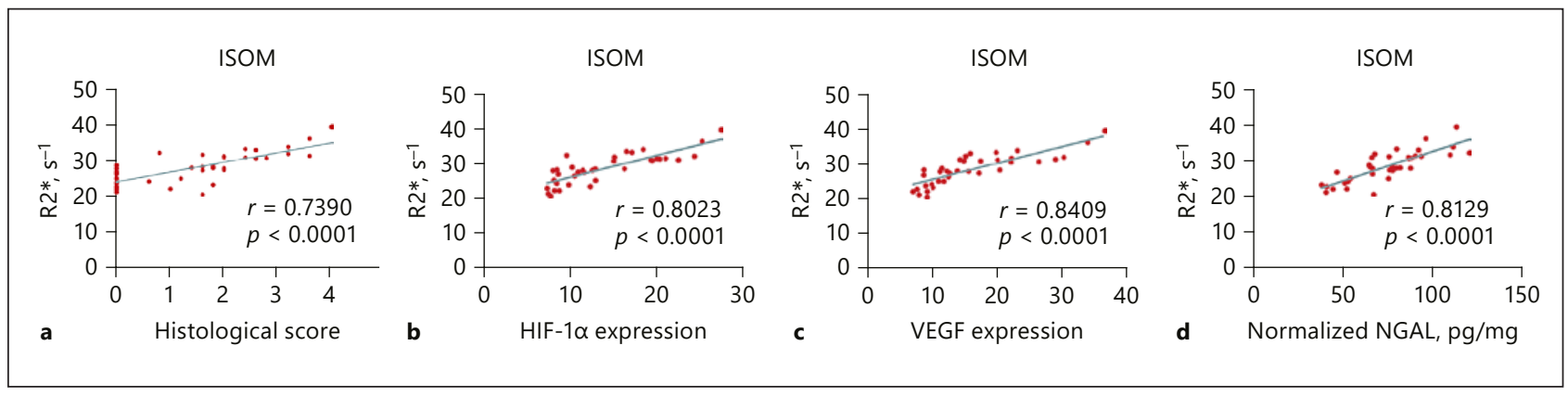

Fig. 12. Correlation between R2* and pathology scores or urinary NGAL. a Correlation between R2* and histological score. b Correlation between R2* and HIF-1 $\alpha$ expression. c Correlation between R2* and VEGF expression. d Correlation between R2* and NGAL.

tubular epithelial cells are exposed to it [32]. Of note, direct contrast-related toxicity is a pivotal criterion for acute renal damage [33].

Our results demonstrated that the peak of blood Scr occurred approximately 3 days after iohexol administration in a rabbit model of CIAKI. However, the change detected at 1 day using urine NGAL was much earlier than that of Scr. fMRI can detect the structural and functional damage of the kidneys before these traditional clinical markers of kidney disease become abnormal. Thus, it is hereby concluded that fMRI combined with urine NGAL is more sensitive than Scr in diagnosing renal impairment after CM injection.

Nevertheless, a few limitations need to be mentioned with regard to the current study. First, due to the poor time efficiency of respiratory-triggered methods, fMRI scanning was performed with a free breathing method. Secondly, the quantitative fMRI multiparameters were calculated as the mean of regions of interest that were manually drawn three times with a uniform size, whereas there is space for measurement bias. Lastly, CM injection affects renal medullary oxygenation and water diffusion [24, 34]; however, we did not evaluate the effect of CM itself on fMRI parameters.

In conclusion, the present study highlights the potential of BOLD imaging combined with IVIM-MRI in examining CIAKI in clinical practice, allowing the measurement of renal oxygenation, hemodynamics, and water diffusion. Increase in iohexol dosage causes more pronounced and prolonged modifications in rabbit kidneys over time. HIF- $1 \alpha$ and VEGF are valuable biomarkers to verify hypoxia in CIAKI as well. uNGAL levels correlate well with fMRI findings; thus, it may be the earliest indicator of renal injury, and future studies could immediately translate the methodology to humans.

\section{Acknowledgments}

The authors thank Guibo Yu for technical assistance (Key Laboratory of Imaging Diagnosis and Interventional Radiology of Liaoning Province, Shenyang, Liaoning, China).

\section{Statement of Ethics}

All the performed experimental protocols were approved by our institute's animal care committee and complied with guidance for the Care and Use of Laboratory Animals (NIH Publication No. 8023, revised 1978). 


\section{Kidney \\ Blood Pressure \\ Research}

\begin{tabular}{l|l}
\hline Kidney Blood Press Res 2019;44:496-512 \\
\hline DOI: 10.1159/000500052 & $\begin{array}{l}\text { @ 2019 The Author(s). Published by S. Karger AG, Basel } \\
\text { www.karger.com/kbr }\end{array}$ \\
\hline
\end{tabular}

Wang et al.: fMRI and Contrast-Induced Acute Kidney Injury

\section{Disclosure Statement}

The authors declare no conflicts of interest.

\section{Funding Sources}

The study was supported by the National Natural Science Foundation of China (Grant 81571635) and Scientific Research Foundation for Advanced Talents, Xiang'an Hospital of Xiamen University (No. PM201809170011).

\section{References}

1 Mozaffarian D, Benjamin EJ, Go AS, Arnett DK, Blaha MJ, Cushman M, et al.; Writing Group Members; American Heart Association Statistics Committee; Stroke Statistics Subcommittee. Executive summary: heart disease and stroke statistics-2016 update: a report from the American Heart Association. Circulation. 2016 Jan; 133(4):447-54.

2 Solomon R. Contrast media: are there differences in nephrotoxicity among contrast media? BioMed Res Int. 2014;2014:934947.

3 Netti GS, Prattichizzo C, Montemurno E, Simone S, Cafiero C, Rascio F, et al. Exposure to low- vs iso-osmolar contrast agents reduces NADPH-dependent reactive oxygen species generation in a cellular model of renal injury. Free Radic Biol Med. 2014 Mar;68:35-42.

4 Liu ZZ, Schmerbach K, Lu Y, Perlewitz A, Nikitina T, Cantow K, et al. Iodinated contrast media cause direct tubular cell damage, leading to oxidative stress, low nitric oxide, and impairment of tubuloglomerular feedback. Am J Physiol Renal Physiol. 2014 Apr;306(8):F864-72.

5 Wang Y, Ren K, Liu Y, Sun WG, Wang JH, Zhang X, et al. Application of BOLD MRI and DTI for the evaluation of renal effect related to viscosity of iodinated contrast agent in a rat model. J Magn Reson Imaging. 2017 Nov; 46(5):1320-31.

6 Ichikawa S, Motosugi U, Ichikawa T, Sano K, Morisaka H, Araki T. Intravoxel incoherent motion imaging of the kidney: alterations in diffusion and perfusion in patients with renal dysfunction. Magn Reson Imaging. 2013 Apr;31(3):414-7.

7 Chen F, Li S, Sun D. Methods of blood oxygen level-dependent magnetic resonance imaging analysis for evaluating renal oxygenation. Kidney Blood Press Res. 2018;43(2):378-88.

8 Pedersen M, Dissing TH, Mørkenborg J, Stødkilde-Jørgensen H, Hansen LH, Pedersen LB, et al. Validation of quantitative BOLD MRI measurements in kidney: application to unilateral ureteral obstruction. Kidney Int. 2005 Jun;67(6):2305-12.

9 Liu M, Guo X, Wang S, Jin M, Wang Y, Li J, et al. BOLD-MRI of breast invasive ductal carcinoma: correlation of R2* value and the expression of HIF-1 $\alpha$. Eur Radiol. 2013 Dec;23(12):3221-7.

10 Sigmund EE, Vivier PH, Sui D, Lamparello NA, Tantillo K, Mikheev A, et al. Intravoxel incoherent motion and diffusion-tensor imaging in renal tissue under hydration and furosemide flow challenges. Radiology. 2012 Jun; 263(3):758-69.

11 Fähling M, Seeliger E, Patzak A, Persson PB. Understanding and preventing contrast-induced acute kidney injury. Nat Rev Nephrol. 2017 Mar;13(3):169-80.

12 Lauver DA, Carey EG, Bergin IL, Lucchesi BR, Gurm HS. Sildenafil citrate for prophylaxis of nephropathy in an animal model of contrast-induced acute kidney injury. PLoS One. 2014 Nov;9(11):e113598.

13 Pettersson G, Towart R, Grant D, Thyberg K, Golman K. The rabbit renal toxicity test: a sensitive in vivo test for the nephrotoxicity of contrast agents. Acad Radiol. 2002 May; 9 Suppl 1:S62-4.

14 Le Bihan D, Breton E, Lallemand D, Aubin ML, Vignaud J, Laval-Jeantet M. Separation of diffusion and perfusion in intravoxel incoherent motion MR imaging. Radiology. 1988 Aug;168(2):497-505.

15 Niles DJ, Gordon JW, Fain SB. Effect of anesthesia on renal R2 * measured by blood oxygen level-dependent MRI. NMR Biomed. 2015 Jul;28(7):811-7.

16 Bhargava AS, Hofmeister R, Siegmund F, Schöbel C, Günzel P. Effect of three non-ionic contrast media on rats and rabbits with regard to renal changes. Interspecies comparison. Arzneimittelforschung. 1990 Jul;40(7): 822-9.

17 Wang JH, Ren K, Sun WG, Zhao L, Zhong HS, Xu K. Effects of iodinated contrast agents on renal oxygenation level determined by blood oxygenation level dependent magnetic resonance imaging in rabbit models of type 1 and type 2 diabetic nephropathy. BMC Nephrol. 2014 Sep;15(1):140.

18 Tsamouri MM, Rapti M, Kouka P, Nepka C, Tsarouhas K, Soumelidis A, et al. Histopathological evaluation and redox assessment in blood and kidney tissues in a rabbit contrast-induced nephrotoxicity model. Food Chem Toxicol. 2017 Oct;108(Pt A):186-93. 
19 Persson PB, Hansell P, Liss P. Pathophysiology of contrast medium-induced nephropathy. Kidney Int. 2005 Jul; 68(1):14-22.

20 Rauch D, Drescher P, Pereira FJ, Knes JM, Will JA, Madsen PO. Comparison of iodinated contrast media-induced renal vasoconstriction in human, rabbit, dog, and pig arteries. Invest Radiol. 1997 Jun;32(6):315-9.

21 Rosenberger C, Heyman SN, Rosen S, Shina A, Goldfarb M, Griethe W, et al. Up-regulation of HIF in experimental acute renal failure: evidence for a protective transcriptional response to hypoxia. Kidney Int. 2005 Feb; 67(2):531-42.

22 Wang J, Zhang Y, Yang X, Wang X, Zhang J, Fang J, et al. Time course study on the effects of iodinated contrast medium on intrarenal water transport function using diffusion-weighted MRI. J Magn Reson Imaging. 2012 May;35(5):1139-44.

23 McMahon GM, Waikar SS. Biomarkers in nephrology: core Curriculum 2013. Am J Kidney Dis. 2013 Jul;62(1): 165-78.

24 Wang YC, Tang A, Chang D, Zhang SJ, Ju S. Significant perturbation in renal functional magnetic resonance imaging parameters and contrast retention for iodixanol compared with iopromide: an experimental study using blood-oxygen-level-dependent/diffusion-weighted magnetic resonance imaging and computed tomography in rats. Invest Radiol. 2014 Nov;49(11):699-706.

25 Liang L, Chen WB, Chan KW, Li YG, Zhang B, Liang CH, et al. Using intravoxel incoherent motion MR imaging to study the renal pathophysiological process of contrast-induced acute kidney injury in rats: comparison with conventional DWI and arterial spin labelling. Eur Radiol. 2016 Jun;26(6):1597-605.

26 Zhang Y, Wang J, Yang X, Wang X, Zhang J, Fang J, et al. The serial effect of iodinated contrast media on renal hemodynamics and oxygenation as evaluated by ASL and BOLD MRI. Contrast Media Mol Imaging. 2012 Jul-Aug; 7(4):418-25.

27 Wybraniec MT, Mizia-Stec K, Więcek A. Contrast-induced acute kidney injury: the dark side of cardiac catheterization. Pol Arch Med Wewn. 2015;125(12):938-49.

28 Kennedy-Lydon TM, Crawford C, Wildman SS, Peppiatt-Wildman CM. Renal pericytes: regulators of medullary blood flow. Acta Physiol (Oxf). 2013 Feb;207(2):212-25.

29 Sendeski MM, Persson AB, Liu ZZ, Busch JF, Weikert S, Persson PB, et al. Iodinated contrast media cause endothelial damage leading to vasoconstriction of human and rat vasa recta. Am J Physiol Renal Physiol. 2012 Dec; 303(12):F1592-8.

30 Mruk B. Renal safety of iodinated contrast media depending on their osmolarity - current outlooks. Pol J Radiol. 2016 Apr;81:157-65.

31 Li LP, Franklin T, Du H, Papadopoulou-Rosenzweig M, Carbray J, Solomon R, et al. Intrarenal oxygenation by blood oxygenation level-dependent MRI in contrast nephropathy model: effect of the viscosity and dose. J Magn Reson Imaging. 2012 Nov;36(5):1162-7.

32 Quintavalle C, Brenca M, De Micco F, Fiore D, Romano S, Romano MF, et al. In vivo and in vitro assessment of pathways involved in contrast media-induced renal cells apoptosis. Cell Death Dis. 2011 May;2(5):e155.

33 Kiss N, Hamar P. Histopathological evaluation of contrast-induced acute kidney injury rodent models. BioMed Res Int. 2016;2016:3763250.

34 Arakelyan K, Cantow K, Hentschel J, Flemming B, Pohlmann A, Ladwig M, et al. Early effects of an X-ray contrast medium on renal T(2)*/T(2) MRI as compared to short-term hyperoxia, hypoxia and aortic occlusion in rats. Acta Physiol (Oxf). 2013 Jun;208(2):202-13. 